OPEN ACCESS

Edited by:

Yoshiyuki lkeda,

Kagoshima University, Japan

Reviewed by:

Vikas Bansal,

Mayo Clinic, United States Akihiro Tokushige,

Kagoshima University, Japan

${ }^{*}$ Correspondence:

Xianwei Zhang

ourpain@163.com

Specialty section:

This article was submitted to

Intensive Care Medicine and

Anesthesiology,

a section of the journal

Frontiers in Medicine

Received: 17 February 2020

Accepted: 16 August 2021

Published: 09 September 2021

Citation:

Zheng H, Duan G, Shen S and

Zhang $X$ (2021) Association of

Nutritional Risk Index With

Postoperative Pain Outcomes in

Elderly Patients Undergoing

Gastrointestinal Surgeries: A

Retrospective Cohort Study.

Front. Med. 8:535627.

doi: 10.3389/fmed.2021.535627

\section{Association of Nutritional Risk Index With Postoperative Pain Outcomes in Elderly Patients Undergoing Gastrointestinal Surgeries: A Retrospective Cohort Study}

\author{
Hua Zheng ${ }^{1}$, Guangyou Duan ${ }^{2}$, Shiqian Shen ${ }^{3}$ and Xianwei Zhang ${ }^{1 *}$ \\ ${ }^{1}$ Department of Anesthesiology, Tongji Hospital, Tongji Medical College, Huazhong University of Science and Technology, \\ Wuhan, China, ${ }^{2}$ Department of Anesthesiology, Second Affiliated Hospital, Chongqing Medical University, Chongqing, China, \\ ${ }^{3}$ MGH Center for Translational Pain Research, Department of Anesthesia, Critical Care and Pain Medicine, Massachusetts \\ General Hospital and Harvard Medical School, Boston, MA, United States
}

Background: Malnutrition is a major health problem, which is common in hospitalized elderly patients and is associated with an increased risk of morbidity and mortality. However, studies on malnutrition and its effect on postoperative pain outcomes in elderly patients have been largely neglected. Here we investigated the relationship between nutritional risk and postoperative pain outcomes in elderly patients.

Methods: Between April 1, 2012, and August 31, 2015, 734 elderly patients ( $\geq 65$ years) who underwent gastrointestinal surgeries were recruited and assigned into two groups according to geriatric nutritional risk index (GNRI). All patients received standard anesthesia procedures and postoperative patient-controlled analgesia for $48 \mathrm{~h}$. The preoperative epidemiology data and postoperative outcome data including pain intensities at rest and movement, the cumulative consumption of analgesics and its common side effects were recorded.

Results: The total number of patients with high nutritional risk (GNRI < 92) was 533 out of 734 (72.62\%). When compared with low nutritional risk individuals (GNRI $\geq$ 92), the incidence of inadequate analgesia was significantly higher in elderly patients with GNRI $<92$ at different time points. In addition, the cumulative consumption of analgesics was also significantly higher in elderly patients with GNRI $<92$ at 0-6h postoperatively. Through logistic regression analysis, high nutritional risk $(\mathrm{OR}=3.113$, 95\% Cl: 1.661-5.834, $P<0.001)$ and female gender $(\mathrm{OR}=0.606,95 \% \mathrm{Cl}$ : 0.394-0.932, $P=0.023$ ) were identified as significant predictors for postoperative inadequate analgesia. Further sensitivity analyses showed high nutritional risk as a predictor for postoperative inadequate analgesia was more prominent in female patients and early elderly patients. Moreover, 88 was determined as an optimal cut-off value of GNRI for postoperative inadequate analgesia using receiver operating characteristic curve analysis. 


\begin{abstract}
Conclusion: High nutritional risk is associated with poor postoperative pain outcomes in gastrointestinal elderly patients. Preoperative nutritional evaluation using simple nutritional screening instruments (e.g., GNRI) with the new suggested cut-off value $(G N R I=88)$ might be included as a standard procedure in routine clinical practice among these patients for postoperative analgesia.
\end{abstract}

Keywords: geriatric nutritional risk index, postoperative pain, postoperative inadequate analgesia, gastrointestinal surgeries, elderly patients

\section{INTRODUCTION}

Population aging is a worldwide phenomenon. It is predicted that persons over 65 years will compose $30 \%$ of the total population by the year 2050 (1). Although these aged individuals are expected to increase demand for surgical treatments (2), management of postoperative pain in elderly patients continues to be a major challenge. It is reported that $\sim 50-75 \%$ of elderly patients experience inadequate postoperative pain relief (3). The under treatment of postoperative pain is associated with serious negative consequences, including increased risk of myocardial, thromboembolic or pulmonary complications, impaired rehabilitation, increased length of hospital stay, increased risk of persistent postoperative pain and elevated mortality rate (4).

The failure to provide appropriate postoperative analgesia in elderly patients is multifactorial. One of the common reasons is inadequate knowledge about physiological or pathophysiological changes and their effects on postoperative pain management in elderly patients (5). As a result of aging processes, elderly patients are at higher risk of malnutrition due to decreased gastric secretions and intestinal motility (6), which has been proven to predict morbidity and mortality among older hospitalized patients $(7,8)$. However, thus far, there have been no studies examining the relationship between nutritional risk and postoperative pain outcomes in elderly patients.

Geriatric nutritional risk index (GNRI) is a nutritional screening and assessment tool created to predict nutritionrelated complications in hospitalized elderly patients (9). A lower value of GNRI indicates a higher nutrition-related risk. In a previous study in elderly patients with acute heart failure, it was found that GNRI $<92$ is associated with poor clinical outcomes (10). In another 3-year follow-up study, a GNRI $<92$ was reported to associated with higher mortality and suggested as a profitable clinical trigger for routine nutritional treatment (11). However, GNRI has not been used to predict postoperative pain outcomes and its optimal cut-off value is unclear.

Accordingly, the main aim of this study was determining whether nutritional risk was associated with postoperative pain outcomes using GNRI and the optimal cutoff value of the GNRI for postoperative inadequate analgesia. Furthermore, we evaluate the influence of nutritional status on postoperative pain outcomes in gastrointestinal elderly patients receiving patient-controlled intravenous analgesia.

\section{MATERIALS AND METHODS \\ Study Design and Data Sources}

Institutional Review Board (IRB) approval for this retrospective cohort study was granted through the Ethic Committee of Tongji Hospital, Tongji Medical College, Huazhong University of Science and Technology (TJ-IRB20190403). The requirement for informed consent from participants was waived under the regulations of IRB. Demographic data (gender, age, weight, height, etc.), preoperative data (comorbidity, serum albumin, etc.) and process data (surgical types, surgical methods, anesthesia techniques, intraoperative medication, analgesia technique, etc.) presented in the current study were extracted from the patients' electronic medical records. Outcome data (pain intensities at rest and movement, cumulative analgesics consumption, side effects of analgesics, etc.) were collected by an acute pain service group at different time points postoperatively (12-14). Approximately $82 \%$ of the patients undergoing gastrointestinal surgeries were included. All data were assessed and edited by two authors (HZ, GD). If a missing data or an extreme value occurred, the relevant raw data were double checked. Participant's name or other form of identification was deleted before analysis. The reporting of this study followed the STROBE (strengthening the reporting of observational studies in epidemiology) (15) and RECORD (reporting of studies conducted using observational routinely collected health data) (16) guidelines.

\section{Participants}

Between April 1, 2012, and August 31, 2015, elderly patients ( $\geq 65$ years) who underwent gastrointestinal surgeries were screened. Inclusion criteria were receiving general anesthesia and postoperative patient-controlled analgesia for $48 \mathrm{~h}$. Exclusion criteria were receiving regional anesthesia, undergone repeat surgery during hospitalization and missing data for any variable.

\section{Exposure of Interest}

The exposure of interest in this study was high nutritional risk. The risk of nutritional status was assessed by the geriatric nutritional risk index (GNRI), which was designed specifically for the hospitalized elderly patients (9). The GNRI was calculated based on the patient's weight, height and serum albumin as follows: GNRI $=[1.489 \times$ albumin $(\mathrm{g} / \mathrm{L})]+[41.7 \times$ 
(weight/WLo)]. The WLo is the ideal weight and was calculated using the Lorentz formula as WLo $=0.75 \times$ height $(\mathrm{cm})-62.5$ for men and WLo $=0.60 \times$ height $(\mathrm{cm})-40$ for women $(17)$. When weight exceeded ideal weight, the ration of weight/WLo was set to 1. Similar to previous studies, the GNRI of 92 was taken as an original cut-off value $(10,11,18)$.

\section{Perioperative Pain Management and Outcome Measures}

All patients were treated according to the standard procedures at Tongji Hospital. In general, anesthesia induction was performed using $0.3-0.6 \mu \mathrm{g} / \mathrm{kg}$ sufentanil, $0.1-0.2 \mathrm{mg} / \mathrm{kg}$ cisatracurium and $1.5-2.5 \mathrm{mg} / \mathrm{kg}$ propofol. Anesthesia was maintained with a combination of sevoflurane (1.0-2.0\%), remifentanil (0.2-0.4 $\left.\mu \mathrm{g} \cdot \mathrm{kg}^{-1} \cdot \mathrm{min}^{-1}\right)$ and propofol $\left(6-10 \mathrm{mg} \cdot \mathrm{kg}^{-1} \cdot \mathrm{h}^{-1}\right)$. At $15 \mathrm{~min}$ prior to the surgery, patients without contraindication were given nonsteroidal anti-inflammatory drugs (NSAIDs, $40 \mathrm{mg}$ parecoxib sodium) and prophylactic antiemetics (dexamethasone $4 \mathrm{mg}$ and/or tropisetron hydrochloride $2 \mathrm{mg}$ ). Immediately after surgery, patient-controlled intravenous analgesia (PCIA) was started with $0.7 \mu \mathrm{g} / \mathrm{mL}$ sufentanil and $4 \mathrm{mg} / \mathrm{mL}$ tramadol using a PCIA pump (BCM, BCDB-150, Shanghai, China). The pump was programmed to use a background infusion at $1-2.5 \mathrm{~mL} / \mathrm{h}$, a bolus dose of $1 \mathrm{~mL}$, a lockout interval of $10 \mathrm{~min}$, and a dose limit of $12 \mathrm{~mL} / \mathrm{h}$.

Patient outcome data were collected by the acute pain service group at $0-6,18-24$, and $42-48 \mathrm{~h}$ postoperatively. Pain intensities at rest and movement were assessed using a $100-\mathrm{mm}$ visual analog scale (VAS, 100 being worst pain imaginable). In addition, cumulative PCIA consumption and the common side effects including postoperative nausea and vomiting, respiratory depression, abdominal distention, pruritus, urinary retention, and dizziness were also recorded. Pain trigger for rescue analgesia was VAS $\geq 40$, which was defined as postoperative inadequate analgesia $(13,19)$. Under these circumstances, Patients without contraindication were given parecoxib sodium and PCIA parameters were upregulated. The primary outcome we used here was postoperative inadequate analgesia. As secondary outcome we investigated cumulative PCIA consumption and the side effects of PCIA.

\section{Statistical Analysis}

In the database, about $8.9 \%$ of data were missed. The missing data arose mainly in variable "postoperative cumulative PCIA consumption" but not in variables included in the regression analysis. Thus, traditional statistical analyses were performed and imputation analyses were not considered. Participants with any missing data were excluded from analysis. Demographic data (e.g., age, weight and height) and postoperative cumulative PCIA consumption were presented as median (interquartile range). These data did not pass the Shapiro-Wilk test for normal distribution and were analyzed by the Mann-Whitney $U$ test. Body mass index (BMI) were presented as mean ( \pm standard deviation) and compared using the $t$ test. Dichotomous data (e.g., gender and postoperative inadequate analgesia) were expressed as absolute number (and \%) and significance was calculated with the chi-square test. To evaluate the role of the preoperative factors in the prediction of postoperative inadequate analgesia during the entire $0-48 \mathrm{~h}$ period, a forward stepwise logistic regression model was applied. Gender, age, BMI, Charlson Comorbidity Index (CCI) score, American society of anesthesiologists (ASA) score, surgical types (gastric or intestinal), surgical methods (endoscopic or non-endoscopic), intraoperative medication and the GNRI were included in the model. Given gender and age were reported to be risk factors for postoperative inadequate analgesia in our previous study (20), sensitivity analyses were further performed in female and male subgroups, as well as in early elderly (age $<75$ years) and late elderly (age $\geq 75$ years) subgroups. A receiver operating characteristic (ROC) curve analysis was used to determine an optimal cut-off value of GNRI for postoperative inadequate analgesia. A $P<0.05$ was considered statistically significant. All of the statistical analyses were performed with SPSS software (version 17.0, SPSS Inc., Chicago, IL, USA).

\section{RESULTS}

\section{Characteristics of the Patients}

During the study period, a total of 806 patients were reviewed. Of them, 59 patients with missing data for any variable, 11 patients receiving regional anesthesia and two patients undergone repeat surgery were excluded. Finally, 734 patients were included in the analysis (Figure 1).

Taking GNRI of 92 as a cut-off point, the total number of patients with high nutritional risk (GNRI < 92) was 533 out of $734(72.62 \%)$. Demographic characteristics of patients were summarized in Table $\mathbf{1}$. The median age of the whole population was 69 (67-74), whereas patients with GNRI $<92$ were slightly older than patients with GNRI $\geq 92(70$ (67-74) vs. 68 (66$73) ; p=0.002)$. Weight (57 (50-65) vs. 63 (58-71); $p<0.001)$, height $(1.64(1.58-1.70)$ vs. $1.65(1.60-1.70) ; p=0.032)$ and BMI $(21.64 \pm 3.60$ vs. $23.70 \pm 2.73$; $p<0.001)$ were lower in patients with GNRI $<92$ compared to patients with GNRI $\geq 92$. In addition, less endoscopic surgeries were performed in patients with GNRI $<92$ than patients with GNRI $\geq 92$ (74.48 vs. $82.09 \%$; $p<0.030)$. Gender, CCI scores, ASA scores, surgical types and intraoperative medication including dexamethasone, parecoxib and sufentanil were not different among groups.

\section{Postoperative Pain Outcomes in Patients With Different Nutritional Risk}

Firstly, we compared postoperative pain outcomes in different nutritional risk groups as classified by the GNRI, which are shown in Table 2. At rest, the incidence of inadequate analgesia of the whole population was $13.62 \%$ at $0-6 \mathrm{~h}, 9.54 \%$ at $18-$ $24 \mathrm{~h}$ and $3.95 \%$ at $42-48 \mathrm{~h}$ postoperatively; the patients with GNRI $<92$ had higher levels of inadequate analgesia at 0$6 \mathrm{~h}$ (16.51 vs. $5.97 \%$; $p<0.001), 18-24$ h (11.82 vs. $3.48 \%$; $p=0.001$ ), but not $42-48 \mathrm{~h}$ postoperatively, in comparison with the patients with GNRI $\geq 92$. On movement, $20.44 \%$ patients at $0-6 \mathrm{~h}, 14.17 \%$ patients at $18-24 \mathrm{~h}$ and $8.99 \%$ patients at $42-48 \mathrm{~h}$ postoperatively presented inadequate analgesia; the incidence of inadequate analgesia in patients with GNRI $<92$ was higher than the patients with GNRI $\geq 92$ at $0-6$ h $(24.02$ vs. $10.95 \%$; 


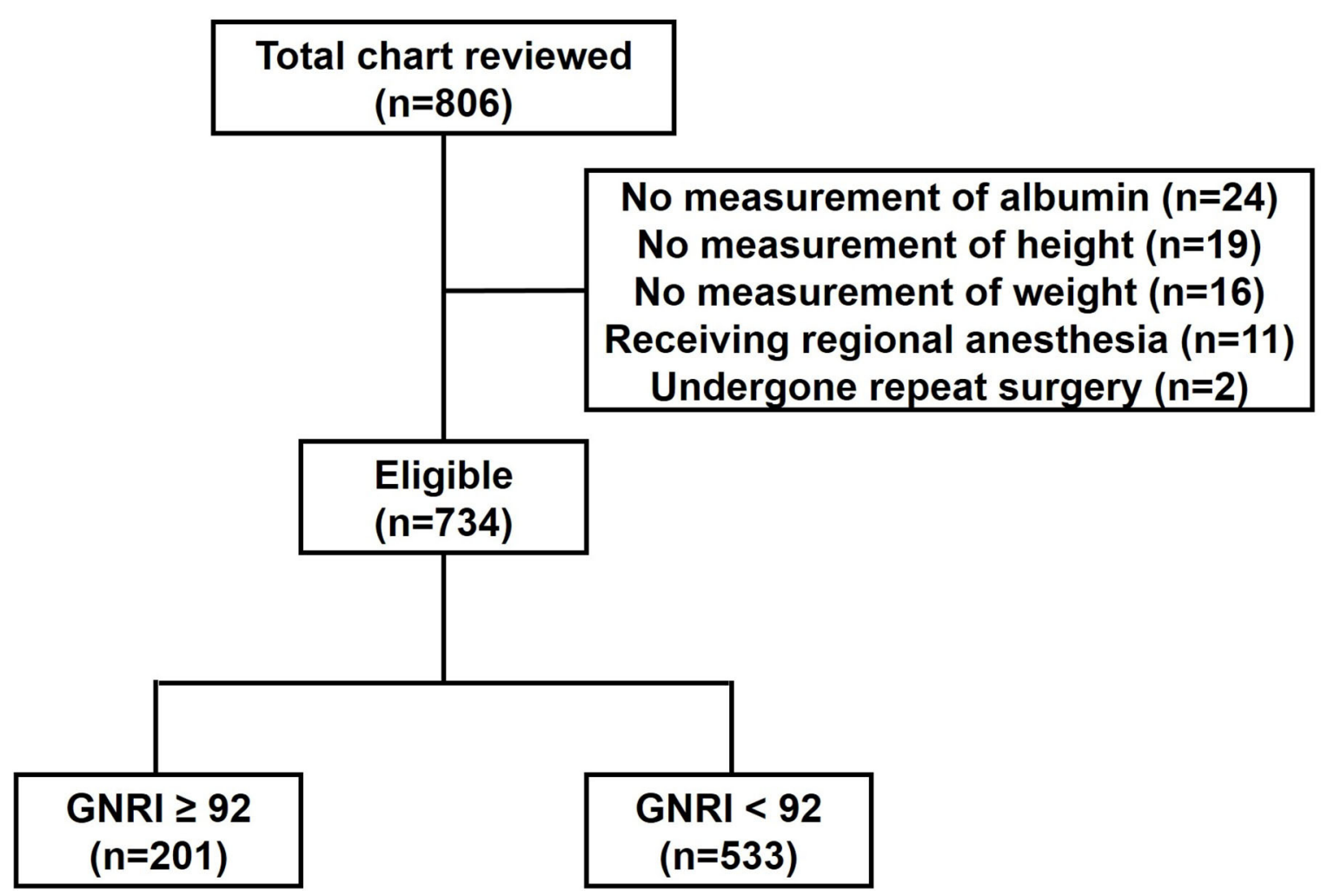

FIGURE 1 | Study flow. GNRI, geriatric nutritional risk index.

TABLE 1 | Comparison of patient characteristics in different nutritional risk groups as classified by the GNRI.

\begin{tabular}{|c|c|c|c|c|}
\hline Characteristic & Total $(n=734)$ & GNRI $\geq 92(n=201)$ & GNRI $<92(n=533)$ & $P$ value \\
\hline Age (years) & $69(67-74)$ & $68(66-73)$ & $70(67-74)$ & 0.002 \\
\hline Height $(\mathrm{m})$ & $1.65(1.59-1.70)$ & $1.65(1.60-1.70)$ & $1.64(1.58-1.70)$ & 0.032 \\
\hline $\mathrm{BMI}\left(\mathrm{kg} / \mathrm{m}^{2}\right)$ & $22.20 \pm 3.51$ & $23.70 \pm 2.73$ & $21.64 \pm 3.60$ & $<0.001$ \\
\hline ASA score $(1 / I / I / I / / V)$ & $\begin{array}{c}262(35.70 \%) / 287 \\
(39.10 \%) / 114(15.53 \%) / \\
71(9.67 \%)\end{array}$ & $\begin{array}{c}82(40.80 \%) / 74 \\
(36.82 \%) / 26(12.94 \%) / \\
19(9.45 \%)\end{array}$ & $\begin{array}{c}180(33.77 \%) / 213 \\
(39.96 \%) / 88(16.51 \%) / \\
52(9.76 \%)\end{array}$ & 0.308 \\
\hline Surgical types (gastric/intestinal) & $415(56.54 \%) / 319(43.46 \%)$ & $118(58.71 \%) / 83(41.29 \%)$ & $297(55.72 \%) / 236$ (44.28\%) & 0.467 \\
\hline Surgical methods (E/non-E) & $562(76.57 \%) / 172(23.43 \%)$ & 165 (82.09\%)/36 (17.91\%) & $397(74.48 \%) / 136$ (25.52\%) & 0.030 \\
\hline Sufentanil $(\mu \mathrm{g})$ & $25(20-30)$ & $25(20-30)$ & $25(20-30)$ & 0.090 \\
\hline
\end{tabular}

Results are presented as median (interquartile range), mean ( \pm standard deviation) or relative numbers [ $n$ (\%)]. Groups were compared by using Mann-Whitney $U$ test, $t$ test or chi-square test. GNRI, geriatric nutritional risk index; BMI, body mass index; CCI, Charlson Comorbidity Index; ASA, American Society of Anesthesiologists; E, endoscopic.

$p<0.001), 18-24 \mathrm{~h}(16.51$ vs. $7.96 \% ; p=0.003)$ and $42-48 \mathrm{~h}$ ( 10.51 vs. $4.98 \% ; p=0.019)$ postoperatively.

In all patients the postoperative cumulative PCIA consumption was $0.15(0.08-0.26) \mathrm{mL} / \mathrm{kg}$ at $0-6 \mathrm{~h}, 0.44$
$(0.27-0.67) \mathrm{mL} / \mathrm{kg}$ at $18-24 \mathrm{k}$ and $0.64(0.41-0.96) \mathrm{mL} / \mathrm{kg}$ at $42-48 \mathrm{~h}$ postoperatively. Patients with GNRI $<92$ received significantly more cumulative PCIA consumption compared to patients with GNRI $\geq 92$ at $0-6 \mathrm{~h}(0.16(0.08-0.27)$ vs. 
TABLE 2 | Comparison of postoperative pain outcomes in different nutritional risk groups as classified by the GNRI.

\begin{tabular}{|c|c|c|c|c|}
\hline Outcome & Total $(n=734)$ & GNRI $\geq 92(n=201)$ & GNRI $<92(n=533)$ & $P$ value \\
\hline \multicolumn{5}{|c|}{ Postoperative inadequate analgesia at rest } \\
\hline $0-6 h$ & $100(13.62 \%)$ & $12(5.97 \%)$ & $88(16.51 \%)$ & $<0.001$ \\
\hline $18-24 h$ & $70(9.54 \%)$ & $7(3.48 \%)$ & $63(11.82 \%)$ & 0.001 \\
\hline $42-48 h$ & $29(3.95 \%)$ & $4(1.99 \%)$ & $25(4.69 \%)$ & 0.094 \\
\hline \multicolumn{5}{|c|}{ Postoperative inadequate analgesia on movement } \\
\hline $0-6 h$ & $150(20.44 \%)$ & $22(10.95 \%)$ & $128(24.02 \%)$ & $<0.001$ \\
\hline $18-24 h$ & $104(14.17 \%)$ & $16(7.96 \%)$ & $88(16.51 \%)$ & 0.003 \\
\hline $42-48 h$ & $66(8.99 \%)$ & $10(4.98 \%)$ & $56(10.51 \%)$ & 0.019 \\
\hline \multicolumn{5}{|c|}{ Postoperative cumulative PCIA consumption (ml/kg) } \\
\hline $0-6 h$ & $0.15(0.08-0.26)$ & $0.15(0.07-0.22)$ & $0.16(0.08-0.27)$ & 0.036 \\
\hline $18-24 h$ & $0.44(0.27-0.67)$ & $0.41(0.28-0.61)$ & $0.46(0.27-0.70)$ & 0.103 \\
\hline $42-48 h$ & $0.64(0.41-0.96)$ & $0.60(0.43-0.92)$ & $0.64(0.40-1.00)$ & 0.246 \\
\hline \multicolumn{5}{|l|}{ Side effects of PCIA } \\
\hline Nausea/vomiting & $101(13.76 \%)$ & $21(10.45 \%)$ & 80 (15.01\%) & 0.110 \\
\hline Dizziness & $36(4.90 \%)$ & $12(5.97 \%)$ & $24(4.50 \%)$ & 0.412 \\
\hline Abdominal distension & 29 (3.95\%) & $6(2.99 \%)$ & $23(4.32 \%)$ & 0.409 \\
\hline Urinary retention & $6(0.82 \%)$ & $2(1.00 \%)$ & $4(0.75 \%)$ & 0.743 \\
\hline Pruritus & $4(0.54 \%)$ & $1(0.50 \%)$ & $3(0.56 \%)$ & 0.915 \\
\hline Respiratory depression & $2(0.27 \%)$ & $0(0.00 \%)$ & $2(0.38 \%)$ & 0.384 \\
\hline
\end{tabular}

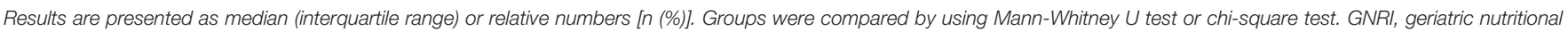
risk index; PCA, Patient-controlled analgesia.

TABLE 3 | Logistic regression analysis investigating possible predictors for postoperative inadequate analgesia.

\begin{tabular}{|c|c|c|c|c|c|}
\hline Population & Predictors & $P$ value & OR & Lower $95 \% \mathrm{Cl}$ & Upper $95 \% \mathrm{Cl}$ \\
\hline Overall $(n=734)$ & Gender & 0.023 & 0.606 & 0.394 & 0.932 \\
\hline Overall $(n=734)$ & GNRI & $<0.001$ & 3.113 & 1.661 & 5.834 \\
\hline Female $(n=262)$ & GNRI & 0.003 & 6.349 & 1.901 & 21.201 \\
\hline Early elderly (Age $<75$ years, $n=587$ ) & GNRI & $<0.001$ & 4.302 & 1.932 & 9.579 \\
\hline
\end{tabular}

GNRI, geriatric nutritional risk index; OR, odds ratio; $\mathrm{Cl}$, confidence interval.

$0.15(0.07-0.22) \mathrm{mL} / \mathrm{kg} ; p=0.036)$, but not at $18-24 \mathrm{~h}$ and 42-48 h postoperatively.

During $48 \mathrm{~h}$ follow-up, postoperative nausea and vomiting was the most common side effect of PCIA with the incidence of $13.76 \%$, followed by dizziness (4.90\%), abdominal distention (3.95\%), urinary retention $(0.82 \%)$, pruritus $(0.54 \%)$ and respiratory depression $(0.27 \%)$. No significant differences were noted regarding the side effects of PCIA among the two groups.

\section{Association of Nutritional Risk With Postoperative Inadequate Analgesia}

Secondly, we performed a logistic regression analyses in order to identify possible predictors for postoperative inadequate analgesia at rest during $48 \mathrm{~h}$ follow-up. The results showed this overall model was significant $(\mathrm{P}<0.001)$. As presented in Table 3, high nutritional risk (OR $=3.113$, 95\% CI: $1.661-$ 5.834, $P<0.001)$ but not age $(P=0.172)$, BMI $(P=0.888)$, CCI score $(P=0.539)$, ASA score $(P=0.701)$, surgical types
$(P=0.814)$, surgical methods $(P=0.859)$ and intraoperative use of dexamethasone $(P=0.698)$, parecoxib $(P=0.282)$ and sufentanil $(P=0.366)$ was identified as a significant predictor for postoperative inadequate analgesia, indicating that the probability of occurrence of postoperative inadequate analgesia in patients with GNRI $<92$ was higher than patients with GNRI $\geq 92$. In addition, The OR for female gender was 0.606 (95\% CI: $0.394-0.932, P=0.023$ ), indicating that, compared with male patients, female patients would have a higher risk to report postoperative inadequate analgesia. Furthermore, sensitivity analyses showed high nutritional risk as a predictor for postoperative inadequate analgesia was more prominent in female patients $(\mathrm{OR}=6.349,95 \% \mathrm{CI}: 1.901-21.201, P=0.003)$ and early elderly patients (OR $=4.302,95 \% \mathrm{CI}: 1.932-9.579$, $P<0.001)$. Collectively, high nutritional risk was associated with postoperative inadequate analgesia in elderly patients after gastrointestinal surgeries, especially in female individuals and early elderly patients. 
Given that GNRI was a predictor for postoperative inadequate analgesia, we used the ROC curve analysis to obtain the area under the curve and an optimal cut-off value of the GNRI. Area under ROC curve was 0.584 (95\% CI: 0.531-0.637, $p=0.007$ ). GNRI $=88$ was determined as an optimal cut-off value with maximum discriminative power. Compared with the original cut-off value of 92 , the new cut-off value of 88 had higher specificity ( 0.465 vs. 0.298$)$ but lower sensitivity ( 0.730 vs. 0.880$)$.

\section{DISCUSSION}

In this large sample of elderly patients after gastrointestinal surgery we showed that GNRI is a significant predictor for postoperative inadequate analgesia at rest during the first $48 \mathrm{~h}$ postoperatively. Elderly patients with lower GNRI values were at higher risk to experience inadequate postoperative pain relief than patients with higher GNRI values. Furthermore, we determined that 88 was an optimal cut-off value of GNRI for postoperative inadequate analgesia.

In the first part of the study, we determined the prevalence of nutrition-related risk according to an original GNRI cut-off value of 92. The results showed that majority (almost three quarters) of elderly patients in the current study were at high nutritional risk. A previous prospective cohort study reports the prevalence of severe and moderate risk of nutritional-related complication in hospitalized elderly patients is $41.2 \%$ (21). A recent retrospective study shows that $61.6 \%$ critical limb ischemia patients are at high nutritional risk (22). Another population-based survey in community-dwelling older persons finds that $69 \%$ of participants are at moderate to high nutritional risk (23). This variability is probably due to the differences of population, measurement instruments and cut-off values. Besides, our results showed that older gastrointestinal patients with high nutritional risk are not uncommon and the prevalence of high nutritional risk has always been underestimated. Thus, using of simple nutritional screening instruments (e.g., GNRI) should be included as a standard procedure in routine clinical practice (17).

Next, we compared patient characteristics and postoperative pain outcomes in different nutritional groups. As expected, we observed that patients with lower GNRI showed significantly higher values of age and lower values of weight, height and BMI. Interestingly, less patients with higher nutritional risk received endoscopic surgeries. The probable reason of this phenomenon might be the higher incidence of cardiopulmonary diseases in patients with higher nutritional risk, which was considered to be a relative contraindication to carbon dioxide pneumoperitoneum during endoscopic surgeries (24). Then, our results showed that the prevalence of postoperative inadequate analgesia at rest and movement was higher in patients with GNRI $<92$ than patients with GNRI $\geq 92$ at different time points. This is consist with a previous study, which shows that pain intensities was higher among patients in low nutritional status than normal patients (25). Another cross-sectional study also shows that the mean nutritional risk score is higher in patients with chronic musculoskeletal pain than patients without chronic musculoskeletal pain (23). Moreover, postoperative cumulative
PCIA consumption was higher in patients with GNRI $<92$ than patients with GNRI $\geq 92$ in the current study. In another word, even the patients with GNRI $<92$ received more analgesics, they still experienced severer postoperative pain. Collectively, high nutritional risk may lead to poor pain management in elderly patients after gastrointestinal surgeries and should not be ignored.

In the second part of the study, we explored whether high nutritional risk is a preoperative factor in the prediction of the primary outcome, postoperative inadequate analgesia. Through regression analysis, high nutritional risk was identified as having a negative effect on postoperative pain. This observation consists with the results of Takahashi et al. (25), who found a correlation between the Nutrition Risk Screening 2002 (NRS 2002) scores and the pain intensities. In community-dwelling older persons, nutritional risk was also reported as being independently associated with chronic musculoskeletal pain (23). The association between high nutritional risk and postoperative inadequate analgesia possibly refers to a systemic inflammatory response, which is triggered by undernutrition and might lead to CNS sensitization and amplification of pain through three pathways (26). Firstly, poor nutrition causes peripheral inflammation, which in turn impacts the CNS (27). Secondly, poor nutrition is associated with cell and tissue damage, which triggers Toll-like receptors activation and central immune signaling events (28). Thirdly, poor nutrition can change gutmicrobiota composition that results in systemic inflammation (29). Additionally, the high levels of anxiety, depression and chronic pain in malnourished individuals, which are all risk factors for poor postoperative pain management, might contribute to postoperative inadequate analgesia as well (3032). In addition, female gender was also identified as a predictor of postoperative inadequate analgesia and women experienced worse postoperative pain relief than men. These results agree with our previous observation in orthopedic patients, which shows that female patients represented severer postoperative pain than male patients (20). Furthermore, high nutritional risk as a predictor for postoperative inadequate analgesia was more prominent in female patients and early elderly patients. Collectively, based on the current study, a better analgesic should be considered for postoperative pain management in elderly patients with high nutritional risk, especially in female patients and early elderly patients.

Next, we determined the optimal value of GNRI using ROC curve analysis. GNRI of 88 was identified as an optimal cut-off value for postoperative inadequate analgesia, which was lower than the original cut-off value of GNRI. The original cut-off value was calculated by using the cut-off values for weight loss and albumin in the elderly (weight/WLo $=0.95$ and albumin $=35$ $\mathrm{g} / \mathrm{L})$ (9). The results in the current study indicate that the optimal cut-off value of GNRI might be different in different conditions. However, the trend is the same that the value of GNRI is lower, the nutrition-related risk is higher. Furthermore, the new cut-off value was more specific but less sensitive than the original cut-off value. Given that inaccurate diagnosis of malnutrition will cause unnecessary treatment and increase the cost of hospitalization, the higher specificity of the new 
cut-off value is extremely important in nutritional assessment (33). Collectively, GNRI $<88$ could be used as a criterion to screen patients' nutrition-related risk of postoperative inadequate analgesia in clinical practice. Early detection of nutrition-related risk before surgeries might contribute to timely nutritional care and the consequent improved postoperative pain outcomes.

Some risk factors for poor postoperative acute pain outcome were identified in previous studies. A large prospective international multicenter database analysis determined 8 risk factors for severe postoperative pain (numeric rating scale $\geq 7$ points) (31). Another meta-analysis of 33 articles identified 9 predictors of poor postoperative pain management (32). However, both studies included more than 50,000 patients with appreciable heterogeneity. Thus, a particular predictor identified might not fit for certain specialties like elderly patients. Through the present analysis focusing on elderly patients, some predictors (e.g., female gender) but not others (such as younger age and higher body mass index) were confirmed. Furthermore, GNRI was added as a novel predictor. These predictors might be useful to stratify inadequate analgesia risk, develop populationspecific clinical care pathways and improve pain outcomes in elderly patients. In elderly patients with high nutritional risk, standardized assessment of nutritional status, adequate implementation of nutritional support and aggressive treatment of postoperative pain should be considered.

The current study has several limitations. Firstly, the results of the current study are based on postoperative data of one single university hospital. However, the single center data may have the strength because of standard treatment, such as similar anesthesia and postoperative analgesic management. Secondly, this is a retrospective cohort study, which has relatively poor control over the exposure factor, covariates, and potential confounders. Therefore, the data obtained in the current study should be cautiously interpreted. Further prospective randomized trials to verify these results are warranted in the future. Thirdly, the new suggested cut-off value of GNRI had relative low sensitivity and specificity. Further studies are needed to evaluate its validity in larger populations. Finally, the generalizability of this study is

\section{REFERENCES}

1. Bettelli G. Anaesthesia for the elderly outpatient: preoperative assessment and evaluation, anaesthetic technique and postoperative pain management. Curr Opin Anaesthesiol. (2010) 23:726-31. doi: 10.1097/ACO.0b013e3283400b6c

2. Etzioni DA, Liu JH, Maggard MA, Ko CY. The aging population and its impact on the surgery workforce. Ann Surg. (2003) 238:1707. doi: 10.1097/01.SLA.0000081085.98792.3d

3. Karani R, Meier DE. Systemic pharmacologic postoperative pain management in the geriatric orthopaedic patient. Clin Orthop Relat Res. (2004) (425):2634. doi: 10.1097/01.blo.0000132403.53010.6f

4. Falzone E, Hoffmann C, Keita H. Postoperative analgesia in elderly patients. Drugs Aging. (2013) 30:81-90. doi: 10.1007/s40266-012-0047-7

5. Herr KA, Garand L. Assessment and measurement of pain in older adults. Clin Geriatr Med. (2001) 17:457-78. doi: 10.1016/S0749-0690(05)70080-X

6. Atkinson TJ, Fudin J, Pandula A, Mirza M. Medication pain management in the elderly: unique and underutilized analgesic treatment options. Clin Ther. (2013) 35:1669-89. doi: 10.1016/j.clinthera.2013.09.008 limited to elderly patients with gastrointestinal surgery. Whether this conclusion is appropriate for patients undergoing other surgeries needs further analysis and studies in the future.

In conclusion, this retrospective cohort study demonstrated that the majority of the hospitalized elderly patients undergoing gastrointestinal surgeries had high nutritionrelated risk using GNRI. In addition, lower GNRI was association with poor postoperative pain outcomes, which indicated the need for early nutritional evaluation and supplementation in elderly patients undergoing gastrointestinal surgeries.

\section{DATA AVAILABILITY STATEMENT}

The datasets generated for this study are available on request to the corresponding author.

\section{ETHICS STATEMENT}

The studies involving human participants were reviewed and approved by the Ethic Committee of Tongji Hospital, Tongji Medical College, Huazhong University of Science and Technology (TJ-IRB20190403). The ethics committee waived the requirement of written informed consent for participation.

\section{AUTHOR CONTRIBUTIONS}

$\mathrm{HZ}$ and $\mathrm{XZ}$ contributed to the conception of the idea and the study design. HZ prepared the data set, performed the analysis, and wrote the manuscript. GD contributed to analysis and interpretation of data. SS and XZ provided intellectual inputs for the project and critical comments on the manuscript. All authors discussed the results and commented on the manuscript.

\section{ACKNOWLEDGMENTS}

We would like to thank Dr. Feng Gao and Ms. Lifang Ren for their support and help in conducting this study.

7. Nicolay RW, Selley RS, Terry MA, Tjong VK. Body mass index as a risk factor for 30-day postoperative complications in knee, hip, and shoulder arthroscopy. Arthroscopy. (2019) 35:874-82. doi: 10.1016/j.arthro.2018.10.108

8. Cho JY, Kim KH, Cho HJ, Lee HY, Choi JO, Jeon ES, et al. Nutritional risk index as a predictor of mortality in acutely decompensated heart failure. PLoS ONE. (2018) 13:e0209088. doi: 10.1371/journal.pone.0209088

9. Bouillanne O, Morineau G, Dupont C, Coulombel I, Vincent JP, Nicolis I, et al. Geriatric Nutritional Risk Index: a new index for evaluating at-risk elderly medical patients. Am J Clin Nutr. (2005) 82:777-83. doi: 10.1093/ajcn/82.4.777

10. Honda Y, Nagai T, Iwakami N, Sugano Y, Honda S, Okada A, et al. Usefulness of geriatric nutritional risk index for assessing nutritional status and its prognostic impact in patients aged $\geq 65$ years with acute heart failure. Am J Cardiol. (2016) 118:550-5. doi: 10.1016/j.amjcard.2016. 05.045

11. Cereda E, Zagami A, Vanotti A, Piffer S, Pedrolli C. Geriatric Nutritional Risk Index and overall-cause mortality prediction in institutionalised elderly: a 3-year survival analysis. Clin Nutr. (2008) 27:717-23. doi: 10.1016/j.clnu.2008.07.005 
12. Zhang Y, Duan G, Guo S, Ying Y, Huang P, Zhang M, et al. To predict sufentanil requirement for postoperative pain control using a real-time method: A prospective observational cohort study. Medicine (Baltimore). (2016) 95:e3915. doi: 10.1097/MD.0000000000003915

13. Duan G, Guo S, Zhang Y, Ying Y, Huang P, Zhang L, et al. Effects of epidemiological factors and pressure pain measurements in predicting postoperative pain: a prospective survey of 1,002 Chinese patients. Pain Physician. (2017) 20:E903-E14.

14. Cao X, Zhang X. Comparison of different sufentanil-tramadol combinations for pain relief within the first 24 hours after cesarean section: a retrospective study. J Pain Res. (2018) 11:2445-51. doi: 10.2147/JPR.S177500

15. Vandenbroucke JP, von Elm E, Altman DG, Gotzsche PC, Mulrow CD, Pocock SJ, et al. Strengthening the Reporting of Observational Studies in Epidemiology (STROBE): explanation and elaboration. PLoS Med. (2007) 4:e297. doi: 10.1371/journal.pmed.0040297

16. Benchimol EI, Smeeth L, Guttmann A, Harron K, Moher D, Petersen I, et al. The REporting of studies Conducted using Observational Routinely-collected health Data (RECORD) statement. PLoS Med. (2015) 12:e1001885. doi: 10.1371/journal.pmed.1001885

17. Abd Aziz NAS, Mohd Fahmi Teng NI, Kamarul Zaman M. Geriatric Nutrition Risk Index is comparable to the mini nutritional assessment for assessing nutritional status in elderly hospitalized patients. Clin Nutr ESPEN. (2019) 29:77-85. doi: 10.1016/j.clnesp.2018.12.002

18. Panichi V, Cupisti A, Rosati A, Di Giorgio A, Scatena A, Menconi O, et al. Geriatric nutritional risk index is a strong predictor of mortality in hemodialysis patients: data from the Riscavid cohort. J Nephrol. (2014) 27:193-201. doi: 10.1007/s40620-013-0033-0

19. Sommer M, de Rijke JM, van Kleef M, Kessels AG, Peters ML, Geurts JW, et al. The prevalence of postoperative pain in a sample of 1490 surgical inpatients. Eur J Anaesthesiol. (2008) 25:267-74. doi: 10.1017/S0265021507003031

20. Zheng H, Schnabel A, Yahiaoui-Doktor M, Meissner W, Van Aken H, Zahn $\mathrm{P}$, et al. Age and preoperative pain are major confounders for sex differences in postoperative pain outcome: A prospective database analysis. PLoS ONE. (2017) 12:e0178659. doi: 10.1371/journal.pone.0178659

21. Abd-El-Gawad WM, Abou-Hashem RM, El Maraghy MO, Amin GE. The validity of Geriatric Nutrition Risk Index: simple tool for prediction of nutritional-related complication of hospitalized elderly patients. Comparison with Mini Nutritional Assessment. Clin Nutr. (2014) 33:1108-16. doi: 10.1016/j.clnu.2013.12.005

22. Luo H, Yang H, Huang B, Yuan D, Zhu J, Zhao J. Geriatric Nutritional Risk Index (GNRI) Independently Predicts Amputation Inchronic Criticallimb Ischemia (CLI). PLoS ONE. (2016) 11:e0152111. doi: 10.1371/journal.pone.0152111

23. Barbara Pereira Costa A, Andrade Carneiro Machado L, Marcos Domingues Dias J, Keller Coelho de. Oliveira A, Ude Viana J, da Silva SL, et al. Nutritional risk is associated with chronic musculoskeletal pain in community-dwelling older persons: The PAINEL study. J Nutr Gerontol Geriatr. (2016) 35:4351. doi: 10.1080/21551197.2015.1125325

24. Galizia G, Prizio G, Lieto E, Castellano P, Pelosio L, Imperatore $\mathrm{V}$, et al. Hemodynamic and pulmonary changes during open, carbon dioxide pneumoperitoneum and abdominal wall-lifting cholecystectomy. A prospective, randomized study. Surg Endosc. (2001) 15:477-83. doi: $10.1007 /$ s004640000343
25. Takahashi H, Chiba T, Tairabune T, Kimura Y, Wakabayashi G, Takahashi $\mathrm{K}$, et al. A retrospective study on the influence of nutritional status on pain management in cancer patients using the transdermal fentanyl patch. Biol Pharm Bull. (2014) 37:853-7. doi: 10.1248/bpb.b13-00717

26. Nijs J, Elma Ö, Yilmaz ST, Mullie P, Vanderweeën L, Clarys P, et al. Nutritional neurobiology and central nervous system sensitisation: missing link in a comprehensive treatment for chronic pain? Br J Anaesth. (2019) 123:53943. doi: 10.1016/j.bja.2019.07.016

27. Schwingshackl L, Hoffmann G. Mediterranean dietary pattern, inflammation and endothelial function: a systematic review and metaanalysis of intervention trials. Nutr Metab Cardiovasc Dis. (2014) 24:929-39. doi: 10.1016/j.numecd.2014.03.003

28. Nicotra L, Loram LC, Watkins LR, Hutchinson MR. Tolllike receptors in chronic pain. Exp Neurol. (2012) 234:31629. doi: 10.1016/j.expneurol.2011.09.038

29. Pimentel GD, Micheletti TO, Pace F, Rosa JC, Santos RV, Lira FS. Gutcentral nervous system axis is a target for nutritional therapies. Nutr J. (2012) 11:22. doi: 10.1186/1475-2891-11-22

30. Chabowski M, Polański J, Jankowska-Polańska B, Janczak D, Rosińczuk J. Is nutritional status associated with the level of anxiety, depression and pain in patients with lung cancer? J Thorac Dis. (2018) 10:230310. doi: 10.21037/jtd.2018.03.108

31. Schnabel A, Yahiaoui-Doktor M, Meissner W, Zahn PK, Pogatzki-Zahn EM. Predicting poor postoperative acute pain outcome in adults: an international, multicentre database analysis of risk factors in 50,005 patients. Pain Rep. (2020) 5:e831. doi: 10.1097/PR9.00000000000 00831

32. Yang MMH, Hartley RL, Leung AA, Ronksley PE, Jetté N, Casha $\mathrm{S}$, et al. Preoperative predictors of poor acute postoperative pain control: a systematic review and meta-analysis. BMJ Open. (2019) 9:e025091. doi: 10.1136/bmjopen-2018-025091

33. Abd Aziz NAS, Teng N, Abdul Hamid MR, Ismail NH. Assessing the nutritional status of hospitalized elderly. Clin Interv Aging. (2017) 12:161525. doi: 10.2147/CIA.S140859

Conflict of Interest: The authors declare that the research was conducted in the absence of any commercial or financial relationships that could be construed as a potential conflict of interest.

Publisher's Note: All claims expressed in this article are solely those of the authors and do not necessarily represent those of their affiliated organizations, or those of the publisher, the editors and the reviewers. Any product that may be evaluated in this article, or claim that may be made by its manufacturer, is not guaranteed or endorsed by the publisher.

Copyright (c) 2021 Zheng, Duan, Shen and Zhang. This is an open-access article distributed under the terms of the Creative Commons Attribution License (CC BY). The use, distribution or reproduction in other forums is permitted, provided the original author(s) and the copyright owner(s) are credited and that the original publication in this journal is cited, in accordance with accepted academic practice. No use, distribution or reproduction is permitted which does not comply with these terms. 